\title{
Non-cell autonomous modulation of tyrosine hydroxylase by HMGB1 released from astrocytes in an acute MPTP-induced Parkinsonian mouse model
}

\author{
Soo Jeong Kim $\mathrm{Kim}^{1,2,3} \cdot$ Min Jeong Ryu ${ }^{1,4} \cdot$ Jeongsu Han ${ }^{1,3} \cdot$ Yunseon Jang ${ }^{1,2,3} \cdot$ Min Joung Lee ${ }^{1,2,3} \cdot$ Xianshu Ju $^{1,2,3}$. \\ Ilhwan Ryu ${ }^{1,2,3} \cdot$ Yu Lim Lee ${ }^{1,2,3} \cdot$ Eungseok $\mathrm{Oh}^{5} \cdot$ Woosuk Chung ${ }^{6,7}$. Jun Young Heo, ${ }^{1,2,3,7} \cdot$ Gi Ryang Kweon ${ }^{1,2,4}$
}

Received: 25 July 2018 / Revised: 20 February 2019 / Accepted: 2 March 2019 / Published online: 1 May 2019

(c) United States \& Canadian Academy of Pathology 2019

\begin{abstract}
High-mobility group box 1 (HMGB1) is actively secreted from inflammatory cells and acts via a non-cell-autonomous mechanism to play an important role in mediating cell proliferation and migration. The HMGB1-RAGE (receptor for advanced glycation end products) axis upregulates tyrosine hydroxylase (TH) expression in response to extracellular insults in dopaminergic neurons in vitro, but little is known about HMGB1 in modulation of dopaminergic neurons in vivo. Here, using immunohistochemistry, we show that HMGB1 and RAGE expression are higher in the nigral area of MPTP (methyl-4phenyl-1,2,3,6-tetrahydropyridine)-treated mice, a toxin-induced Parkinsonian mouse model, compared with saline-treated controls. HMGB1 was predominantly localized to astrocytes and may affect neighboring dopaminergic neurons in the MPTP mouse model, owing to co-localization of RAGE in these TH-positive cells. In addition, MPTP induced a decrease in TH expression, an effect that was potentiated by inhibition of c-Jun N-terminal kinase (JNK) or RAGE. Moreover, stereotaxic injection of recombinant HMGB1 attenuated the MPTP-induced reduction of TH in a Parkinsonian mouse model. Collectively, our results suggest that an increase of HMGB1, released from astrocytes, upregulates TH expression in an acute MPTP-induced Parkinsonian mouse model, thereby maintaining dopaminergic neuronal functions.
\end{abstract}

These authors contributed equally: Soo Jeong Kim, min Jeong Ryu

Supplementary information The online version of this article (https:// doi.org/10.1038/s41374-019-0254-5) contains supplementary material, which is available to authorized users.

Jun Young Heo

junyoung3@gmail.com

$\triangle$ Gi Ryang Kweon

mitochondria@cnu.ac.kr

1 Department of Biochemistry, College of medicine, Chungnam National University, Jung-gu Munhwa-ro 266, Daejeon 35015, Republic of Korea

2 Department of Medical science, College of medicine, Chungnam National University, Jung-gu Munhwa-ro 266, Daejeon 35015, Republic of Korea

3 Infection Control Convergence Research Center, College of medicine, Chungnam National University, Jung-gu Munhwa-ro

\section{Introduction}

High-mobility group box 1 (HMGB1) is a nonhistone DNA-binding nuclear protein. In the nucleus, HMGB1 acts to sustain nucleosome formation, chromosomal stability, DNA repair, and telomere maintenance through a cellautonomous mechanism [1]. In addition to its role in the nucleus, HMGB1 is reported to be secreted via both active and passive pathways: passively via burst release from

266, Daejeon 35015, Republic of Korea

4 Research Institute for Medical Science, College of medicine, Chungnam National University, Jung-gu Munhwa-ro 266, Daejeon 35015, Republic of Korea

5 Department of Neurology, Chungnam National University Hospital, Jung-gu Munhwa-ro 282, Daejeon 35015, Republic of Korea

6 Department of Anesthesiology and Pain Medicine, Chungnam National University Hospital, Jung-gu Munhwa-ro 282, Daejeon 35015, Republic of Korea

7 Brain research Institute, College of medicine, Chungnam National University, Jung-gu Munhwa-ro 266, Daejeon 35015, Republic of Korea 
necrotic cells, and actively from myeloid lineage cells in response to various stimuli, such as endotoxin, tumor necrosis factor alpha (TNF $\alpha$ ), and reactive oxygen species (ROS) [2]. However, a fuller understanding of the physiological role of secreted HMGB1 in cell-to-cell interactions requires an experimental strategy using in vivo models.

In mouse models of focal cerebral ischemia, it has been shown that inhibition of HMGB1 secretion by neutralizing monoclonal antibodies reduces the severity of lesions and suppresses microglial activation [3]. In contrast to the potential deleterious effects of HMGB1 on neurons, enhanced release of HMGB1 from astrocytes has been reported to promote neurite outgrowth and endothelial cell activation in the context of neurovascular remodeling after stroke-induced damage in mice [4]. Furthermore, overexpression of HMGB1 has been shown to repair cerebellar mitochondrial DNA damage in ATXN1 (ataxin 1) knock-in mice [5]. In terms of neurodegenerative diseases, such as Parkinson's disease (PD), it has been shown that HMGB1 is induced in glial cells in the 6-hydroxydopamine (6-OHDA) rat model of PD and is detected in cerebrospinal fluid (CSF) and serum of PD patients. HMGB1 also preferentially binds to aggregated $\alpha$-synuclein and is present in the $\alpha$-synuclein filament containing Lewy bodies [6]. Moreover, a neutralizing antibody against HMGB1 was shown to attenuate dopaminergic neuron loss in animal models of PD [7]. However, previous studies have not identified a non-cellautonomous intracellular signaling mechanism for HMGB1 actions in PD.

Receptors for HMGB1 include Toll-like receptor $2 / 4$ (TLR2/4), CXC chemokine receptor 4 (CXCR4), receptor for advanced glycation end product (RAGE), and antigen complex 1 (MAC-1) [8]. Upon binding to specific receptors, which vary depending on the redox form of HMGB1, HMGB1 induces different responses in target cells, including inflammation, proliferation/migration, and resolution of inflammation [9-11]. In addition to the redox state of HMGB1, induction of target receptors in specific disease states increases the possibility of crosstalk between HMGB1 and different receptors. Unlike TLR2/4 and CXCR4 and MAC-1, RAGE is known to be an inducible receptor in progressive degenerative diseases, and its activation by HMGB1 binding has been implicated in sterile inflammation as well as cancer, diabetes, and Alzheimer's disease [12, 13].

Given the numerous cell types in the brain microenvironment that affect the maintenance of neuronal circuitry, we sought to determine the role of the HMGB1RAGE axis in dopaminergic neurons in vivo in an acute methyl-4-phenyl-1,2,3,6-tetrahydropyridine (MPTP) Parkinsonian mouse model. We further found that recombinant HMGB1 prevents the reduction in $\mathrm{TH}$ in the SN4741 dopaminergic neuronal cell line induced by the active MPTP metabolite, $\mathrm{MPP}^{+}$[14]. In accordance with our previous reports, we here demonstrate that recombinant HMGB1 (rHMGB1) signals through RAGE to upregulate tyrosine hydroxylase (TH) expression via a c-Jun N-terminal kinase (JNK)-dependent mechanism in MPTP model mice.

\section{Materials and methods}

\section{Chemicals, reagents, and antibodies}

1-methyl-4-phenyl-1,2,3,6-tetrahydropyridine (MPTP, M0896), 1-methyl-4-phenylpyridinium (MPP $\left.{ }^{+}, \mathrm{D} 048\right)$, and MG-132 (M8699) were purchased from Sigma-Aldrich (St. Louis, MO, USA). Lipofectamine 2000 (11668027) was purchased from Thermo-Fisher Scientific (CA, USA). AntiRAGE (rabbit polyclonal, ab3611) was purchased from Abcam (Cambridge, MA, USA). Anti-phospho JNK (rabbit polyclonal, \#9251) and anti-total JNK (rabbit polyclonal, \#9252) were purchased from Cell Signaling Technology (Beverly, MA, USA). Anti-GFAP (chicken polyclonal, ab4674) and anti-HMGB1 (rabbit polyclonal, ab18256), were purchased from Abcam (Cambridge, MA, USA). AntiIba1 (goat polyclonal, NB100-1028) was purchased from Novus (Littleton, CO, USA). Anti-TH (rabbit polyclonal, AB152) was purchased from Millipore (Temecula, CA, USA). Anti-PARP (rabbit polyclonal, sc-7150), anti-actin (rabbit polyclonal, sc-1616), anti-ubiquitination (mouse monoclonal, sc-8017), and Protein A/G PLUS-agarose beads (sc-2003) were purchased from Santa Cruz Biotechnology (Santa Cruz, CA, USA). Recombinant human HMGB1 (1690-HMB) was purchased from R\&D Systems (Minneapolis, USA). RAGE antagonist/inhibitor (553030) was purchased from Calbiochem (Temecula, CA, USA). JNK inhibitor SP600125 (S5567) was purchased from Sigma-Aldrich.

\section{Cell lines and culture conditions}

The dopaminergic neuronal progenitor cell line (SN4741) was cultured as described before [15]. SN4741 cells were grown in RF medium containing Dulbecco's modified Eagle's medium (DMEM) (Thermo-Fisher Scientific Inc., Waltham, MA, USA) supplemented with $10 \%$ fetal bovine serum (FBS) (Thermo-Fisher Scientific Inc.), $1 \%$ glucose (Amresco, Radnor, PA, USA), 1\% penicillin-streptomycin, and L-glutamine ( $2 \mathrm{mM})$ (Thermo-Fisher Scientific Inc.) at $33{ }^{\circ} \mathrm{C}$ with $5 \% \mathrm{CO}_{2}$. To western blot the $\mathrm{TH}$ protein, SN4741 cells were differentiated by transferring the culture of SN4741 cells to RF culture medium containing 0.5-1\% fetal calf serum (FCS) (Thermo-Fisher Scientific Inc.) at 5\% 
$\mathrm{CO}_{2}$ at a nonpermissive temperature $\left(37^{\circ} \mathrm{C}\right)$ as described previously [15]. The microglial cell line, BV-2 was maintained in DMEM containing $10 \%$ FBS and $1 \%$ penicillin-streptomycin at $37^{\circ} \mathrm{C}$ and $5 \% \quad \mathrm{CO}_{2}$. Human glioblastoma cells (U87MG) were purchased from the Korean Cell Line Bank (Seoul National University, Korea) and cultured in Minimum Essential Medium (MEM) (Thermo-Fisher Scientific Inc.) supplemented with $10 \%$ FBS and $1 \%$ penicillin-streptomycin at $37^{\circ} \mathrm{C}$ in a humidified atmosphere of $5 \% \mathrm{CO}_{2}$ without differentiation.

\section{Primary cortical astrocytes}

Primary cortical astrocytes were prepared from postnatal day 0-3 (PND 0-3) C57BL/6 mice. Cells were placed in DMEM supplemented with $25 \mathrm{mM}$ glucose, $10 \%$ heatinactivated horse serum (Sigma-Aldrich, St. Louis, MO, USA), 2 mM L-glutamine, $10 \%$ heat-inactivated FBS, and $1000 \mathrm{U} / \mathrm{ml}$ penicillin-streptomycin. Cultures were maintained at $37{ }^{\circ} \mathrm{C}$ in a humidified $5 \% \mathrm{CO}_{2}$ incubator. On the third day of culture, the cells were washed vigorously by repeated pipetting, and the medium was exchanged.

\section{Animals and MPTP administration}

Male C57Bl/6 mice (21-25 g; age 8 weeks) were given intraperitoneal injections of sterile saline or MPTP administered as four times injections of $20 \mathrm{mg} / \mathrm{kg}$ at $2-\mathrm{h}$ intervals [16] and were killed at selected time points after the last injection (1, 3, 5, and 7 days). Control animals were injected with an equal volume of $0.9 \%$ sterile saline. Mice were divided into four groups $(n=4)$ : group 1, vehicle control; group 2, vehicle and MPTP $20 \mathrm{mg} / \mathrm{kg}$ i.p.; groups 3 and 4 were treated with MPTP $20 \mathrm{mg} / \mathrm{kg}$ i.p. with RAGE inhibitor and JNK inhibitor at a dose of $20 \mathrm{mg} / \mathrm{kg}$ twice (during the first and the last MPTP injection). Mice were killed at day 1 after the final injection of MPTP. For the study of stereotaxic injections, mice were divided into three groups $(n=6)$ : group 1, vehicle i.p. and stereotaxic injections with saline control; group 2, MPTP 20 $\mathrm{mg} / \mathrm{kg}$ i.p. and stereotaxic injections with saline; group 3, MPTP $20 \mathrm{mg} / \mathrm{kg}$ i.p. and stereotaxic injections with recombinant HMGB1 at a dose of $100 \mathrm{ng}$. After 5 days of MPTP injection, stereotaxic injections were performed with saline or recombinant HMGB1. Mice were killed at 1 day after stereotaxic injections. All mouse experiments were performed in the animal facility according to institutional guidelines (SOP, standard operating procedure), and the experimental protocols were approved by the institutional review board of the Chungnam National University (CNU-00356).

\section{Immunohistochemistry and immunofluorescence}

Saline and MPTP-injected mouse were perfused with perfusion solution and post fixated with $4 \%$ paraformaldehyde for 1 day. The perfusion solution was made with $\mathrm{NaCl}$, $\mathrm{NaNO}_{3}$ (Sigma-Aldrich), and heparin in dissolving distilled water. Cryoprotected up to $30 \%$ sucrose buffer, the brains were cut coronally into $30-\mu \mathrm{m}$ thickness. Brain slices were fixed with tissue stock solution and rinsed three times in phosphate-buffered saline (PBS, pH 7.4). In total, 0.3\% Triton X-100 and 2\% donkey serum (Gene Tex, Irvine, CA, USA) in PBS was used for blocking for $90 \mathrm{~min}$, and then brain slices were incubated with primary antibodies against anti-GFAP, anti-HMGB1, anti-Tyrosine hydroxylase, antiRAGE, and anti-Ibal at $4{ }^{\circ} \mathrm{C}$ overnight. After that, for immunofluorescence, brain slices were incubated with secondary antibodies at RT for $90 \mathrm{~min}$ : anti-chicken Alexa Fluor 488, anti-rabbit Alexa Fluor 594, and anti-goat Alexa Fluor 647. For immunohistochemistry, brain slices were incubated with rabbit secondary antibodies (Dako EnVision $^{+}$system-HRP, USA) for 90 min, and then, reacting with $\mathrm{DAB}^{+}$substrate buffer. After mounting by a fluorescent mounting medium (Dako North America Inc., USA) on cover slides, immunofluorescent images were acquired using Olympus ${ }^{\mathrm{TM}}$ confocal microscope (Olympus).

\section{Stereological cell counts}

The total number of TH-positive neurons within the SN were calculated based on the optical fractionator technique using a bright-field microscope (Olympus Optical, BX51, Tokyo, Japan). The immunostained TH neurons were counted within the boundaries of the optical fractionator. The estimated total number of TH-positive neurons were calculated according to the optical fractionator software (MBF Bioscience, Williston, VA, USA). We analyzed more than 300 points for every part of each sample.

\section{Real-time polymerase chain reaction (PCR) analysis}

Total RNA was extracted using Trizol reagent according to the manufacturer's instructions, and real-time quantitative PCR was performed using cDNA, 7500 Real-Time PCR (Applied Biosystems), and SYBR Green PCR Master Mix (iCycler iQ Real-Time PCR Detection System; Bio-Rad, Hercules, CA, USA) detection. All primers were designed by the Primer3 program which is used widely for designing PCR primers to amplify fragments. Relative gene expression was quantified and normalized with respect to the $18 \mathrm{~s}$ ribosomal RNA (housekeeping gene as endogenous control) and the following primers: HMGB1 (forward) $55^{\prime}$-cagctggaggatgtgtctca-3', (reverse) $5^{\prime}$-ggcatgacggatgtactgtg- $3^{\prime} .18 \mathrm{~s}$ 
rRNA (forward) 5'-ctg gttgatcctgccagtag-3', (reverse) 5'cgaccaaaggaaccataact- $3^{\prime}$.

\section{Western blot analysis}

Cells and brain tissues were extracted with RIPA lysis buffer [5 mM EDTA, $100 \mathrm{mM}$ Tris- $\mathrm{HCl}$ (pH 8.5), $200 \mathrm{mM}$ $\mathrm{NaCl}$, and $0.2 \%$ SDS with phosphatase and protease inhibitor cocktail]. After centrifugation at $15,000 \times g$ for $20 \mathrm{~min}$ at $4{ }^{\circ} \mathrm{C}$, supernatants were collected. Protein levels were measured using Bradford method [17]. Isolated protein $(20 \mu \mathrm{g})$ was resolved using 9 12\% SDS-PAGE and transferred onto polyvinylidene fluoride membranes (PVDF) at $100 \mathrm{~V}$ for $2 \mathrm{~h}$, which were blocked with $3 \%$ BSA in TBST [10 mM Tris-HCL (pH 7.6), $150 \mathrm{mM} \mathrm{NaCl,} \mathrm{and} 0.1 \%$ Tween 20] for $30 \mathrm{~min}-1 \mathrm{~h}$. The membranes were incubated overnight at $4{ }^{\circ} \mathrm{C}$ with primary antibodies and their bindings were detected using the appropriate secondary antibodies coupled with horseradish peroxidase, as described by the manufacturer's instructions. Finally, the antibody-labeled proteins were detected using the ECL chemiluminescence system (WEST-ZOL plus, iNtRON BioTechnology, Korea).

\section{Immunoprecipitations}

For immunoprecipitations, SN4741 cells were treated with $100 \mathrm{ng} / \mathrm{ml}$ of recombinant human for $4 \mathrm{~h}$ and $10 \mu \mathrm{M}$ of MG132 was added for $2 \mathrm{~h}$. The cells were washed twice with cold PBS and lysed with lysis buffer containing Tris- $\mathrm{Cl}(\mathrm{pH}$ 7.9, $20 \mathrm{mM}), \mathrm{NaCl}(120 \mathrm{mM})$, Triton X-100 (0.5\%), EDTA $(2.5 \mathrm{mM})$, and DTT $(2 \mathrm{mM})$ in the presence of protease and phosphatase inhibitor cocktails. The cell lysates were incubated with an anti-TH antibody at $4{ }^{\circ} \mathrm{C}$ for $16 \mathrm{~h}$ and then treated with Protein A/G PLUS-agarose beads for $2 \mathrm{~h}$ at $4{ }^{\circ} \mathrm{C}$. The precipitates were eluted from the beads by boiling with the addition of SDS sample buffer. Whole-cell lysates and eluted samples were performed with western blot analysis with anti-TH, anti-Ub, and anti-Actin antibodies.

\section{ELISA}

A supplementary medium from U87MG cells and primary cortical astrocytes with treatment of $\mathrm{MPP}^{+}$dose was collected and measured by enzyme-linked immunosorbent assay (ELISA) (IBL, Toronto, Ontario, Canada), which detects mouse HMGB1, according to the manufacturer's instructions.

\section{CCK-8 assay}

Cell viability was determined by the Cell Counting Kit-8 (CCK-8), which was purchased from Dojindo (Rockville,
MD, USA). The CCK- 8 assay was used to measure cytotoxicity, and U87MG cells were plated at $1 \times 10^{4}$ cells per well in 96-well culture plates at $37^{\circ} \mathrm{C}$ for $24 \mathrm{~h}$ under conditions with a range of concentrations (0-6 mM). CCK-8 solution was added to each well, and the absorbance was measured at 450-nm wavelength by MultiSkan Ascent microplate spectrophotometer (Thermo-Fisher Scientific Inc., CA, USA).

\section{Stereotaxic injections}

Male C57BL/6 mice (21-25 g, 8-week old) were injected intraperitoneally (i.p.) with MPTP $(20 \mathrm{mg} / \mathrm{kg}$ ) four times at 2-h intervals for 5 days, with or without co-injection of recombinant HMGB1 (rHMGB1); saline-injected mice were used as controls. After the last injection of MPTP on day 5, mice in the MPTP + rHMGB1 group were anesthetized with an i.p. injection of Avertin (T48402; Sigma-Aldrich, St. Louis, MO, USA) and then stereotaxically injected with $100 \mathrm{ng}$ of rHMGB1. A single needle inserted into the right brain was used to target the striatum at AP: $+0.19 \mathrm{~mm}$; ML: $-0.15 \mathrm{~mm}$ to bregma DV: $-2.6 \mathrm{~mm}$ to the skull [18]. rHMGB1 was injected via a Hamilton syringe at a rate of $0.2 \mu \mathrm{l} / \mathrm{min}(2 \mu \mathrm{l}$ in total per site); the needle was left in place for at least $10 \mathrm{~min}$ at each target site to prevent backflow through the needle track. Twenty-four hours after recovering from surgery, animals were killed for immunohistochemistry and western blot analysis.

\section{TH promoter activity analysis}

HEK293 cells were seeded into a 96-well plate and transiently transfected with TH promoter-EGFP vector (pAAV2.5-THpGFP was a gift from Kwang-Soo Kim) (Addgene plasmid \# 80336; http://n2t.net/addgene:80336; RRID:Addgene_80336) [19] with Lipofectamine 2000. After $24 \mathrm{~h}$, cells were treated with $100 \mathrm{ng} / \mathrm{ml}$ of rHMGB1 with/without $20 \mu \mathrm{M}$ SP600125 or $100 \mathrm{nM}$ of RAGE inhibitor for $6 \mathrm{~h}$. The fluorescence was measured at excitation and emission wavelengths of 485 and $535 \mathrm{~nm}$, respectively, using a microplate fluorometer (Berthold Technologies, Bad Wildbad, Germany).

\section{Statistical analysis}

All results were shown as mean values+SEM (error bars). Statistical analysis was conducted according to each experiment, and Tukey's post hoc analysis was performed by one-way analysis of variance (ANOVA) and two-tailed unpaired Student's t test using GraphPad Instat (GraphPad Software Inc., San Diego, CA, USA). P-value $<0.05$ was considered statistically significant $(* P<0.05$, $* * P<0.01$, and $* * * P<0.001)$. 


\section{Results}

\section{HMGB1 is increased in MPTP-treated mice and is secreted from astrocytes}

The acute MPTP PD model has been well characterized in terms of the MPTP dose and time required to observe decreases in the number of TH-positive cells in the nigrostriatal pathway of the mouse brain [20]. In this acute model, four injections of $20 \mathrm{mg} / \mathrm{kg}$ MPTP administered at $2 \mathrm{~h}$ four times during the day showed dopaminergic neurodegeneration and deletion of striatal dopamine after administration of MPTP for 7 days (Supplementary Fig. S1a-e) [16, 21]. To assess the physiological role of HMGB1 in TH regulation in the acute MPTP mouse model, we examined the HMGB1 expression pattern in the nigral pathway by immunohistochemistry using a specific HMGB1 antibody. After 7 days of MPTP injections, HMGB1 staining density was increased more than twofold in the region surrounding the substantia nigra pars compacta (SNpc) compared with that in the salinetreated group (Supplementary Fig. 2a, b). In contrast, there were no changes in the HMGB1 staining pattern in the striatal area at this time point (Supplementary Fig. 2c, d). Consistent with these immunostaining results, western blotting showed that expression levels of HMGB1 protein were more than 1.5fold higher in the SNpc region after 7 days of MPTP injection, whereas expression levels were unchanged in the striatum (Supplementary Fig. 2e-h).

The brain parenchyma is composed of numerous cell types [22], a complicating factor that was not applicable in our previous in vitro studies [14]. To verify a non-cellautonomous mechanism for HMGB1, we thus first need to identify the cell types which expressed higher levels of HMGB1 in MPTP-treated mice. To this end, we performed double-immunofluorescence staining of the $\mathrm{SNpc}$ area for HMGB1 and the astrocytic marker, glial fibrillary acidic protein (GFAP), or dopaminergic neuronal marker, TH. Numerous GFAP-positive astrocytes were detected in the SNpc area of MPTP-injected mice, whereas the number of TH-positive dopaminergic neurons was decreased in these mice. In control (saline-treated) mice, HMGB1 was colocalized with both TH-positive dopaminergic neurons and GFAP-positive astrocytes, but MPTP injection enhanced the intensity of HMGB1 staining in GFAP-positive astrocytes and increased the number of HMGB1/GFAP double-positive astrocytes (Fig. 1a-c and Supplementary Fig. 3a). Because a previous study reported HMGB1 immunostaining in microglia in a drug-induced PD mouse model [7, 23], we performed double-immunofluorescence staining for HMGB1 and ionized calcium-binding adapter molecule 1 (Iba1), a microglia marker. HMGB1 and Iba1 co-expression in the SNpc area was rare in controls and exhibited a tendency toward induction in the acute MPTP mouse model (Supplementary Fig. 3b-d).
To confirm the astrocyte specificity of HMGB1 secretion, we assessed HMGB1 expression in the U87MG human astrocyte-like glioblastoma cell line, BV-2 microglial cell line, and SN4741 dopaminergic neuronal progenitor cell line. Interestingly, administration of 1methyl-4-phenylpyridinium $\left(\mathrm{MPP}^{+}\right)$, the active metabolite of MPTP, had no effect on HMGB1 expression in BV-2 or SN4741 cells (Fig. 1d, e), but induced a concentration- and time-dependent increase in HMGB1 mRNA and protein expression in U87MG cells (Fig. 1f-h). To assess HMGB1 secretion by astrocytes, we also measured the HMGB1 concentration in U87MG and primary cultured astrocytes cell-conditioned medium using enzyme-linked immunosorbent assays (ELISA) after treatment with $\mathrm{MPP}^{+}$. Because treatment with $3 \mathrm{mM} \mathrm{MPP}^{+}$reduced cell viability (Supplementary Fig. 4a), we tested HMGB1 secretion at lower concentrations of $\mathrm{MPP}^{+}$, and found a significant concentration-dependent increase (Fig. 1i, j), indicating that HMGB1 is actively secreted by these astrocytes. Taken together, our results indicate that HMGB1 is upregulated in astrocytes in vivo in MPTP-treated mice, and is secreted by astrocytes in vitro in response to treatment with the MPTPactive metabolite, $\mathrm{MPP}^{+}$.

\section{RAGE expression is increased in the nigrostriatal pathway and co-localizes with dopaminergic neurons in acute MPTP PD model mice}

Next, we investigated the expression of RAGE, which is expressed by neurons and endothelial cells, and is known to serve as an HMGB1 receptor [24]. Immunostaining with an anti-RAGE antibody revealed that RAGE expression was increased in the SNpc and striatal areas of MPTP-treated mice compared with controls (Fig. 2a-d). Consistent with these immunostaining results, immunoblotting showed that RAGE expression was increased in the nigrostriatal pathway (Fig. 2e-h). After 7 days of MPTP injection, HMGB1 and RAGE were concomitantly induced in the SNpc of PD model mice; moreover, unlike HMGB1, RAGE expression was also elevated in the striatal region. Next, we used immunohistochemistry to identify the localization of RAGE in order to determine the site of the HMGB1-RAGE axis. Interestingly, RAGE signals showed no overlap with astrocytes or microglial cells (Supplementary Fig. 5a-d). Instead, we found that RAGE was co-localized with THpositive dopaminergic neurons, and that the percentage of TH-positive neurons expressing RAGE was increased by MPTP injection (Fig. 2i, j). RAGE staining intensity was also higher in TH-positive cells of MPTP-treated mice (Fig. 2k). Taken together, these findings indicate that MPTP increases both the number of RAGE-expressing cells and the intensity of RAGE staining in TH-positive dopaminergic neurons. 
a

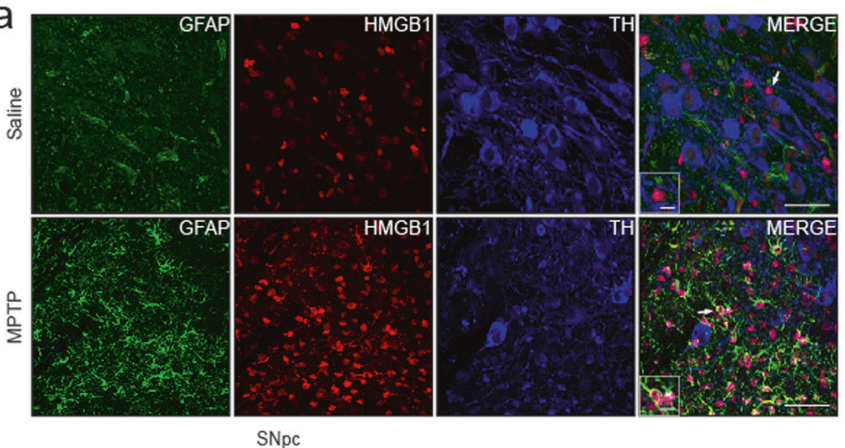

b

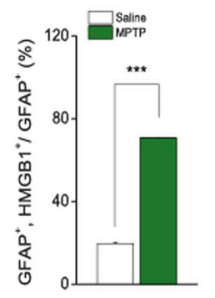

C

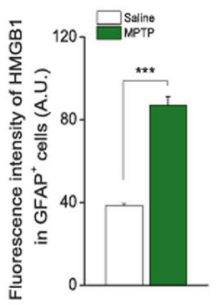

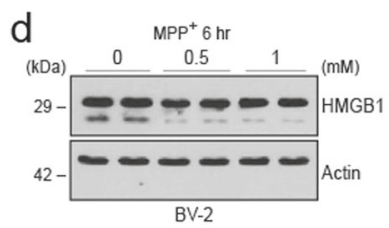

h

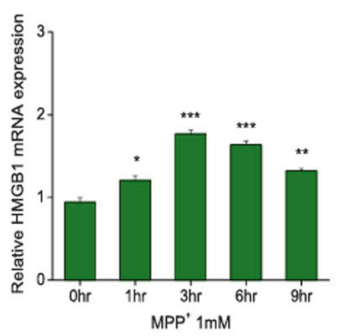

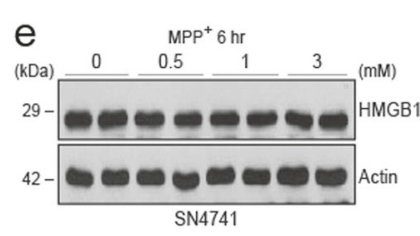

i

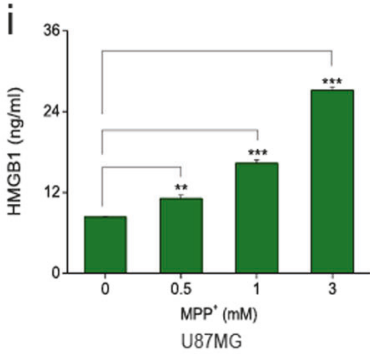

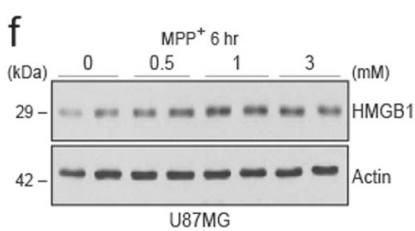
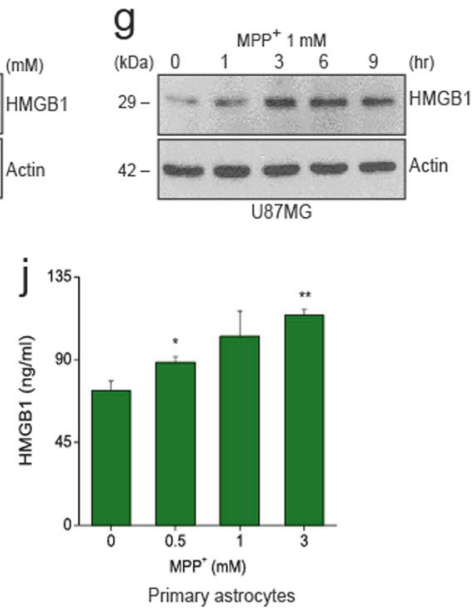

Fig. 1 Increased HMGB1 expression in the SNpc and glial cells following MPTP and $\mathrm{MPP}^{+}$treatment, respectively. a Brain SNpc regions were immunostained for the astrocyte marker GFAP (green), HMGB1 (red), and the dopaminergic neuron marker TH (blue). Scale bars: $16.6 \mu \mathrm{m}$. b, c Percent of GFAP-positive cells co-expressing HMGB1 (b), and HMGB1 fluorescence intensity in GFAP-positive cells (c) ( $n=7 /$ group). d-f HMGB1 protein expression levels in BV-2 cells (d), SN4741 cells (e), and U87MG cells (f) following treatment with $\mathrm{MPP}^{+}(0-3 \mathrm{mM})$ for $6 \mathrm{~h}$. Actin was used as a loading control. $\mathbf{g}, \mathbf{h}$

\section{Inhibition of JNK or RAGE exacerbates the abrupt loss of TH expression in the nigrostriatal pathway in the acute MPTP mouse model}

We previously demonstrated that HMGB1-RAGE signaling acts through the JNK pathway to induce TH expression in dopaminergic neurons. To investigate this relationship in vivo, we assessed TH expression in the acute MPTP mouse model by co-injecting (i.p.) a JNK inhibitor (JNKi or SP660125) or a RAGE inhibitor (RAGEi) together with MPTP. The optimal injection time point was determined by analyzing HMGB1 and TH expression in acute MPTPtreated mice over time. HMGB1 expression was increased in MPTP-treated mice relative to controls 1 day post injection in the striatum and 3 days post injection in the SNpc area (Supplementary Fig. 6a-d). In contrast, HMGB1 expression was not changed in the hippocampal area (Supplementary Fig. 6e, f), which does not contain dopaminergic neurons, and was thus regarded as a control region
U87MG cells were treated with $1 \mathrm{mM} \mathrm{MPP}^{+}$for $0-9 \mathrm{~h}$, after which there were HMGB1 protein levels (g) and mRNA (h) in cells. Supernatants of U87MG cells (i) and primary cultured astrocytes (j) were harvested and subjected to ELISA analysis after $6 \mathrm{~h}$ of $\mathrm{MPP}^{+}$ treatment. Data represent means +SEM (error bars) from three independent experiments performed under the same conditions $(n=6$; $* p<0.05, * * p<0.01$, and $* * * p<0.001$ compared with controls; oneway ANOVA followed by Tukey's post hoc analysis)

[25]. Because HMGB1 upregulation was detected 1 day after MPTP injection in the striatum (Supplementary Fig. 6a), we injected JNK and RAGE inhibitors on the same day as and 1 day after MPTP injections, and performed western blot analyses on the SNpc and striatum. Whereas treatment with MPTP decreased TH protein expression to 55 and $62 \%$ of controls in samples from the SNpc and striatum, respectively, as determined by densitometric analysis of western blot bands, treatment with a JNK or RAGE inhibitor potentiated the MPTP-induced decrease in TH protein expression in the $\mathrm{SNpc}$ and striatum, reducing $\mathrm{TH}$ band density in samples from the $\mathrm{SNpc}$ to $33 \%$ in the MPTP + JNKi group and $28 \%$ in the MPTP + RAGEi group; in samples from the striatum, the corresponding values relative to controls were $23 \%$ in the MPTP + JNKi group and $43 \%$ in MPTP + RAGEi group (Fig. 3a-d). Collectively, these results suggest that HMGB1 attenuates the decrease in TH expression observed in the acute MPTP mouse model in a RAGE- and JNK-dependent manner. 


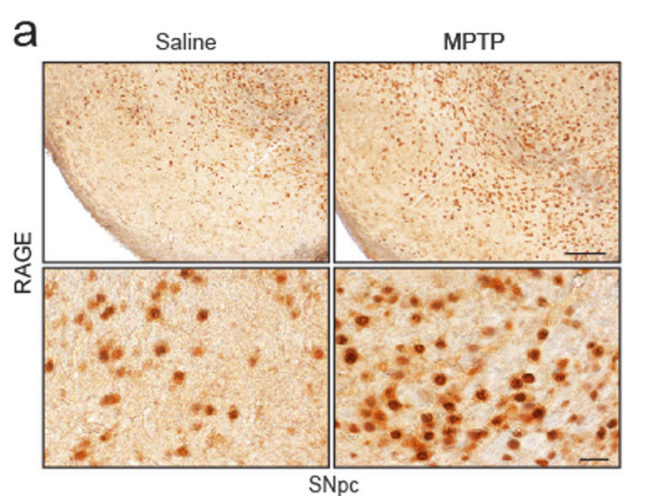

b

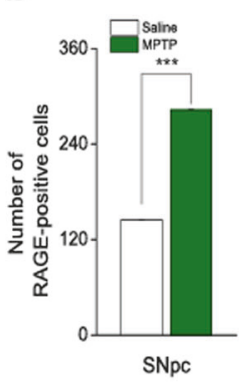

e
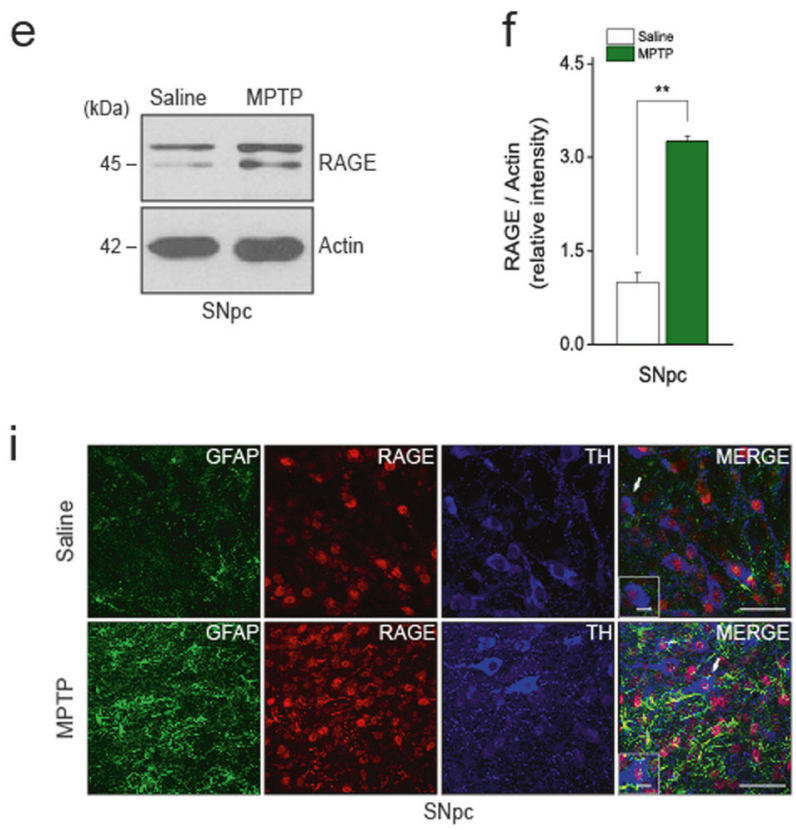

Fig. 2 Increased RAGE expression in the nigrostriatal pathway in the MPTP Parkinsonian mouse model. a, c Photomicrographs showing RAGE immunostaining in the SNpc at low magnification (upper panel; scale bars, $100 \mu \mathrm{m}$ ) and high magnification (lower panel; scale bars, $25 \mu \mathrm{m})(\mathbf{a})$, and in the striatum at low magnification (scale bars, $250 \mu \mathrm{m})(\mathbf{c})(n=7 / g r o u p)$. b, d Number of RAGE-positive cells in the SNpc (b), and optical density of RAGE in the striatum, determined using Image $\mathbf{J}$ software (d) $(n=7 /$ group). $* * * p<0.001$ (two-tailed unpaired Student's $t$-test). e, $\mathbf{g}$ Protein levels of RAGE in SNpc and striatum were determined by western blotting after administration of

\section{Administration of rHMGB1 attenuates the loss of TH expression in the acute MPTP mouse model}

Because blocking of HMGB1 or RAGE signaling potentiated the decrease in $\mathrm{TH}$ expression, we sought to determine whether administration of HMGB1 to MPTP-treated mice exerted the opposite effect. Because HMGB1 was temporarily induced from 1 to 3 days after MPTP injection in the striatum and returned to levels observed in salinetreated groups after 5 days (Supplementary Fig. 6a), we performed stereotaxic injection experiments after 5 days of MPTP treatment (Fig. 4a). Under these conditions,
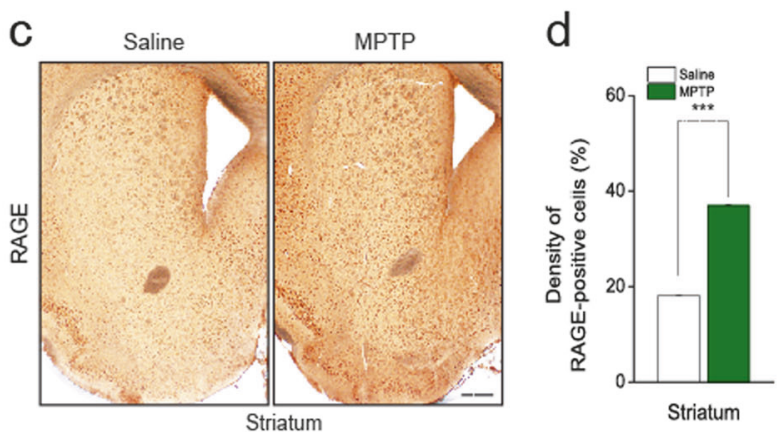

g
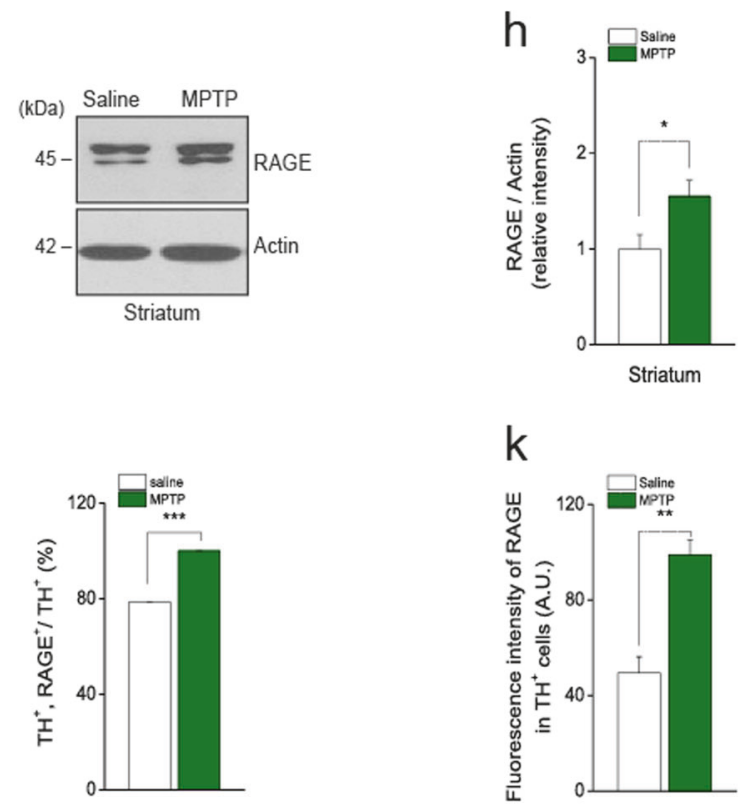

MPTP. Actin was used as a loading control. f, h Relative intensity of RAGE in panels of $\mathbf{e}$ and $\mathbf{g}$, normalized with respect to actin ( $n=8$ / group). ${ }^{*} p<0.05,{ }^{*} p<0.01$ (two-tailed unpaired Student's $t$-test). i The SNpc was immunostained for the astrocyte marker GFAP (green), RAGE (red), and dopaminergic neuron marker TH (blue). Scale bars: $16.6 \mu \mathrm{m}$. j, k Percentage of TH-positive neurons expressing RAGE (j), and fluorescence intensity of RAGE staining in TH-positive cells (k). Data represent means +SEM (error bars) from three experiments performed under the same conditions ( $n=7 /$ group). $* * p<0.01, * * * p<0.001$ (two-tailed unpaired Student's $t$-test)

treatment with rHMBG1 for $24 \mathrm{~h}$ rescued the MPTPinduced reduction in TH staining intensity in the striatum. Whereas the density of TH-positive fibers in the striatum was reduced to $44 \%$ in the MPTP group, TH-positive fiber density was increased to $79 \%$ in the MPTP + rHMGB 1 group, a value comparable with that in the saline-treated group ( $82 \%$ ) (Fig. $4 \mathrm{~b}, \mathrm{c}$ ). To confirm TH protein levels in the striatum, we performed immunoblotting using an antiTH antibody. Consistent with immunostaining results, western blotting showed that the reduction in TH protein levels induced by MPTP treatment was rescued by rHMGB1 injection (Fig. 4d, e). 
Fig. $3 \mathrm{TH}$ and p-JNK expression patterns in the presence and absence of JNK or RAGE inhibitors in the acute MPTP mouse model. a, c Protein levels of $\mathrm{p}-\mathrm{JNK}$ and $\mathrm{TH}$ were determined by western blotting in the striatum (a) and $\mathrm{SNpc}(\mathbf{c})$ after administration of MPTP, with/without an inhibitor of JNK or RAGE ( $n=8 /$ group). Actin was used as a loading control. b, d Quantification of p-JNK and $\mathrm{TH}$ band densities in samples from the striatum (b) and $\mathrm{SNpc}$ (d). Data represent means + SEM from three experiments performed under the same conditions $(* p<0.05$, *** $p<$ 0.001 compared with saline controls; one-way ANOVA using Tukey's test). JNKi, JNK inhibitor (SP660125); RAGEi, RAGE inhibitor; ns, not significant
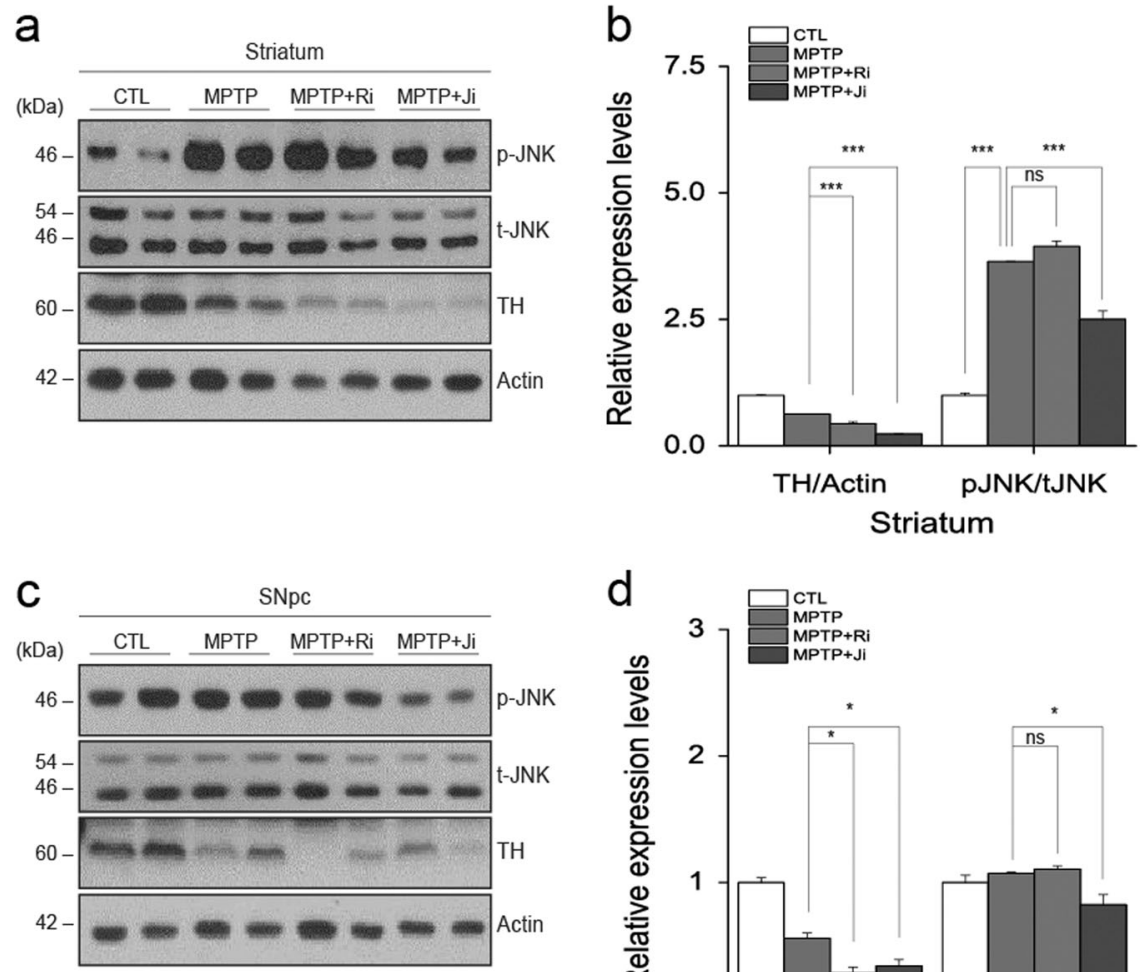

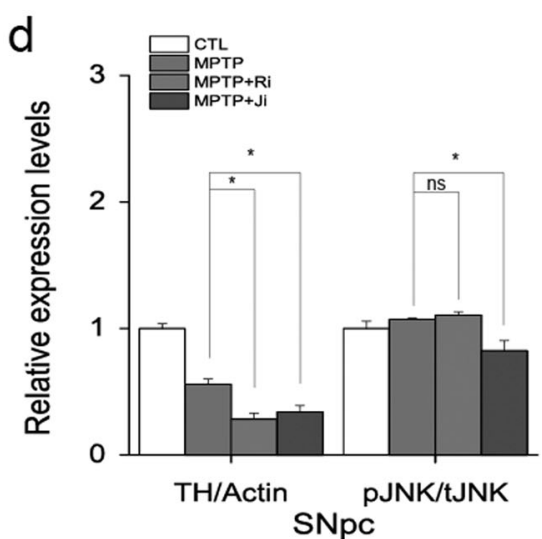

Taken together, these findings indicate that maintained levels of HMGB 1 protein in the striatum mitigate the abrupt, MPTP-induced reduction in TH protein levels in vivo.

\section{Discussion}

The main pathology of PD is dopaminergic neuron dysfunction, mediated by $\alpha$-synuclein deposition, and neuroinflammation, mediated by astrocyte-microglia interactions [26, 27]. Paradoxically, neuroinflammation may also be regarded as a defense reaction that serves to protect neurons against toxins and other noxious agents or insults [28]. Therefore, the induction of inflammation in PD is thought to be a double-edged sword, as it triggers the release of various molecular mediators, such as cytokines and DAMPs, from reactive glial cells [29]. A previous study suggested that neuron-released $\alpha$-synuclein acts through TLR2-dependent microglial activation to cause the noncell-autonomous loss of dopaminergic neurons, as also supported by the MPTP-intoxication model in mice $[30,31]$. In addition to neuronal loss caused by the release of DAMPs, we found that HMGB1, released from reactive astrocytes in the acute period of MPTP intoxication in mice, affects the maintenance of dopaminergic neuronal function through modulation of TH expression.
Astrocytes undergo morphological changes during activation by stimuli, such as oxidative stress [32], and can release DAMPs, which initiate immune responses in the absence of infectious agents. In PD, these DAMPs include S100 $\beta$, heat-shock proteins, and HMGB1 [33]. S100 $\beta$, which is mainly expressed in astrocytes in the central nervous system (CNS) under healthy conditions [34], was found to be unaltered in the serum of PD patients compared with age-matched controls. However, the HMGB1 concentration in CSF is elevated in PD patients, and this DAMP has been shown to be relevant to the neurotoxic mechanism [35-37]. Induction of HMGB1 has been reported in 6OHDA rat and subacute MPTP mouse models [7, 23]. Here, we show that HMGB1 expression was increased 1 or 3 days after MPTP treatment, but later returns back to the basal level. This transient increase of HMGB1 expression by MPTP in our mouse model is understandable, since the halflife of $\mathrm{MPP}^{+}$concentration of mice treated with MPTP was within $20 \mathrm{~h}[38,39]$. In addition, HMGB1 is localized to the $\mathrm{SNpc}$ region in the acute MPTP mouse model, especially in reactive astrocytes, dopaminergic neurons, and microglia. However, induction of HMGB1 was only detected in GFAPpositive astrocytes, not in Iba1-positive microglia, in the acute MPTP mouse model. Therefore, we propose that astrocyte-secreted HMGB1 might be an important mediator that sustains PD neurodegeneration. 
Fig. $4 \mathrm{TH}$ expression patterns following rHMGB1 injection in the acute MPTP mouse model. a rHMGB1 treatment schedule in the MPTP mouse model. b TH immunostaining in the striatum on day 6 after MPTP injection, with or without rHMGB1 injection. Scale bars, $250 \mu \mathrm{m}$. c Striatal fiber loss in MPTP model mice, expressed as a percentage, with or without rHMGB1 treatment $(n=8 /$ group). d Western blot analysis of $\mathrm{TH}$ in the striatum. e Quantification of TH band densities. Data represent means + SEM from three experiments performed under the same conditions $(* p<0.05, * * * p<$ 0.001 compared with saline controls; one-way ANOVA using Tukey's test)
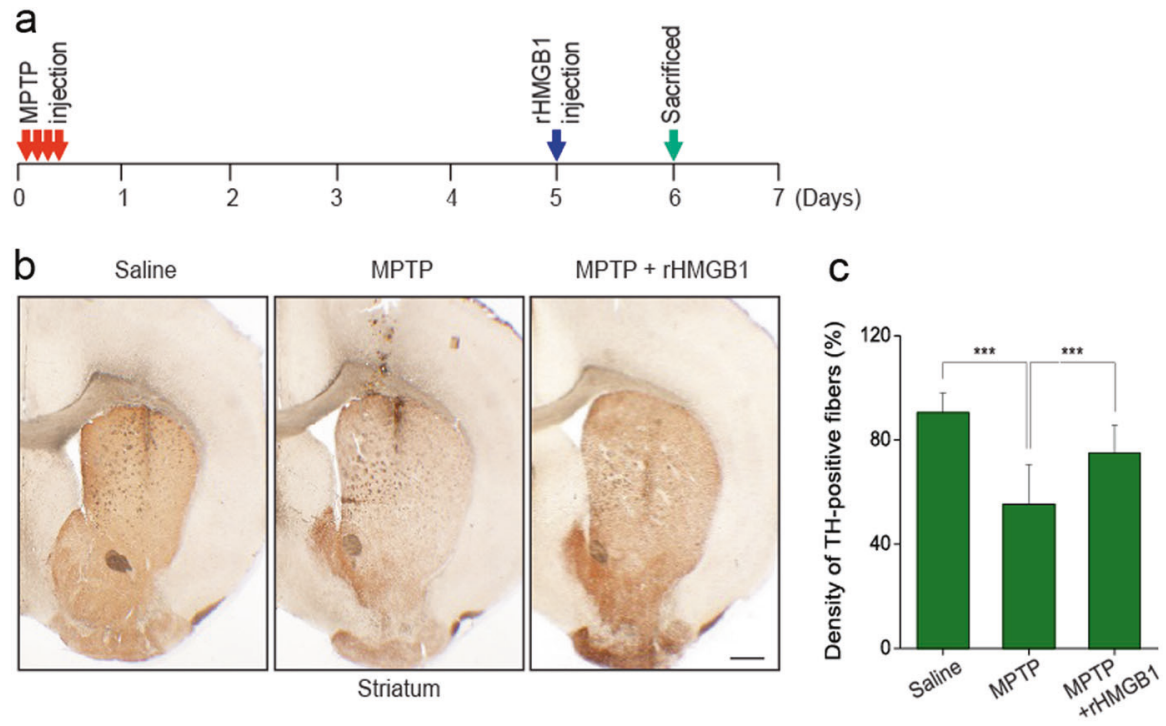

d

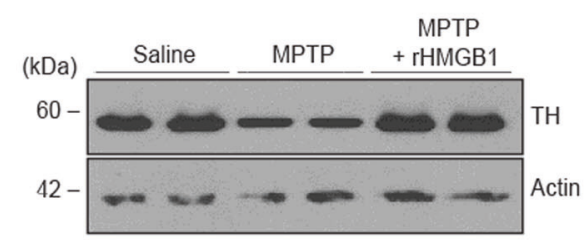

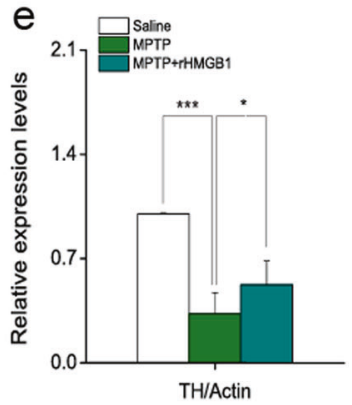

RAGE, first identified and described for its ability to bind to advanced glycation end products (AGE), is a transmembrane receptor of the immunoglobulin superfamily. Several studies have shown that ligand-RAGE interactions mediate cell migration and upregulation of proinflammatory and prothrombotic molecules in mononuclear cells, macrophages, and endothelial cells [40]. RAGE has also been linked to several chronic diseases that are thought to result from RAGE/NF- $\kappa \mathrm{B}$ signaling-dependent inflammation. In a triple-transgenic mouse model of Alzheimer's disease and the subacute MPTP mouse model, expression of RAGE is increased in neurons and astrocytes [41]. In this study, we also found induction of RAGE expression and colocalization with $\mathrm{TH}$-positive neurons in the $\mathrm{SNpc}$ region in the acute MPTP mouse model.

The interaction of HMGB1 with RAGE can activate two major pathways, one comprising CDC42/Rac and the other involving diverse mitogen-activated protein kinases (MAPKs), including p38 MAPK, p44/42, and SAPK/JNK, ultimately leading to cytoskeletal changes and $\mathrm{NF}-\kappa \mathrm{B}$ activation, respectively [42, 43]. Moreover, the HMGB1RAGE pathway is known to induce inflammatory responses via JNK signaling in normal human bronchial epithelial cells [44]. In our previous study, we found that treatment with rHMGB1 activated the JNK pathway in dopaminergic neurons [14]. However, although inhibition of RAGE or JNK further potentiated the MPTP-induced decrease in TH expression, pharmacological inhibition of RAGE did not inhibit JNK in the MPTP mouse model. This observation suggests that further studies are needed to identify potential additional microenvironmental mediators of $\mathrm{TH}$ expression induced by the HMGB1-RAGE axis in the MPTP mouse model.

In a recent study, Santoro et al. confirmed that MPTPinduced dopaminergic cell death was attenuated by HMGB1neutralizing antibody with intraperitoneal injection prior to treatment with MPTP and every third day afterward in a subacute MPTP-induced mice model [7]. Sasaki et al. also reported attenuated PD behavioral symptoms after intravenous HMGB1 antibody administration, immediately 6 and $24 \mathrm{~h}$ after 6-OHDA injection in rats [23]. Both studies injected HMGB1 antibodies into the systemic circulation, not by direct stereotaxic injection into the striatal or SNpc region. Since peripheral lymphocyte infiltration into the brain contributes to neurodegeneration in the Parkinson's disease mouse model [45], systemic injection of HMGB1-neutralizing antibody can prevent the infiltration of lymphocytes via deactivation of lymphocyte proliferation. Moreover, intraperitoneal injection of glycyrrhizin (HMGB1 inhibitor) can attenuate the severity of experimental autoimmune encephalomyelitis through reducing 
inflammatory cell infiltration, decreasing inflammatory cytokines [46]. Therefore, the therapeutic approach by using systemic injection of an anti-HMGB1 antibody in PD implicates in the inactivation of inflammatory cells, prevention of lymphocyte infiltration, and neuroinflammation. Different with a systemic effect of an anti-HMGB1 antibody, our focus on this study is the investigation of secreted HMGB1 function from astrocytes in the brain; therefore, we injected the rHMGB1 with stereotaxic surgery, which was performed at the time when HMGB1 expression was reduced in the MPTP mouse model, and found that $\mathrm{TH}$ expression was improved in the striatum. To confirm the direct regulation of $\mathrm{TH}$ induction by HMGB1, promoter activity assay was assessed using TH promoter-EGFP plasmid in HEK293 cells, and ubiquitination assay was performed in SN4741 cells. As a result, green fluorescence intensity is increased in HEK293 cells after treating with rHMGB1, which is inhibited by treating with JNK and RAGE inhibitors (Supplementary Fig. 7a). In addition, rHMGB1 treatment increased TH immunoblot activity, but regardless of rHMGB1 treatment, no band reacting with Ub antibody was detected in lysates immunoprecipitated through the TH antibody (Supplementary Fig. 7b). We identify that HMGB1 promotes TH expression transcriptionally, without altering the ubiquitination of the $\mathrm{TH}$ protein. In conclusion, the difference in the results of HMGB1 injection comes from the difference of the injection protocol, which are the number of injection trials and injection sites. We suggest that elicitation of HMGB1 function is needed to separate between a systemic and brain direct effect in further study.

Previous reports have suggested several possible mechanisms underlying altered TH expression, including receptor-mediated cell-autonomous activity and synaptic input [47]. In the rat nigrostriatal system, it has also been reported that $\mathrm{TH}$ protein upregulation may reflect a compensatory response of dopaminergic neurons that serves to enhance their biosynthetic capacity and increase the efficiency of dopamine neurotransmission after traumatic brain injury [48]. Taken together, our data suggest that HMGB1 acts as a compensatory factor that attenuates the MPTPinduced abrupt loss of $\mathrm{TH}$ expression and dopaminergic neuronal dysfunction in vivo in the acute MPTP-induced Parkinsonian mouse model.

Acknowledgements This work was funded by the National Research Foundation of Korea (NRF) grant, the Ministry of Science and ICT (MSIT) (2016R1A2B4010398), (2017R1A5A2015385) and the Ministry of Education (2014R1A6A1029617), (2016R1D1A1B03932766), and by the research fund of the Chungnam National University.

\section{Compliance with ethical standards}

Conflict of interest The authors declare that they have no conflict of interest.
Publisher's note: Springer Nature remains neutral with regard to jurisdictional claims in published maps and institutional affiliations.

\section{References}

1. VanPatten S, Al-Abed Y. High mobility group Box-1 (HMGb1): current wisdom and advancement as a potential drug target. J Med Chem. 2018;61:5093-107.

2. Tang D, Kang R, Zeh HJ 3rd, Lotze MT. High-mobility group box 1, oxidative stress, and disease. Antioxid Redox Signal. 2011;14:1315-35.

3. Liu K, Mori S, Takahashi HK, Tomono Y, Wake H, Kanke T, et al. Anti-high mobility group box 1 monoclonal antibody ameliorates brain infarction induced by transient ischemia in rats. FASEB J. 2007;21:3904-16.

4. Hayakawa K, Pham LD, Katusic ZS, Arai K, Lo EH. Astrocytic high-mobility group box 1 promotes endothelial progenitor cellmediated neurovascular remodeling during stroke recovery. Proc Natl Acad Sci USA. 2012;109:7505-10.

5. Ito H, Fujita K, Tagawa K, Chen X, Homma H, Sasabe T, et al. HMGB1 facilitates repair of mitochondrial DNA damage and extends the lifespan of mutant ataxin-1 knock-in mice. EMBO Mol Med. 2015;7:78-101.

6. Lindersson EK, Hojrup P, Gai WP, LockerD, Martin D, Jensen $\mathrm{PH}$. alpha-Synuclein filaments bind the transcriptional regulator HMGB-1. Neuroreport. 2004;15:2735-9.

7. Santoro M, Maetzler W, Stathakos P, Martin HL, Hobert MA, Rattay TW, et al. In-vivo evidence that high mobility group box 1 exerts deleterious effects in the 1-methyl-4-phenyl1,2,3,6-tetrahydropyridine model and Parkinson's disease which can be attenuated by glycyrrhizin. Neurobiol Dis. 2016;91:59-68.

8. Gao HM, Zhou H, Zhang F, Wilson BC, Kam W, Hong JS. HMGB1 acts on microglia Mac1 to mediate chronic neuroinflammation that drives progressive neurodegeneration. J Neurosci. 2011;31:1081-92.

9. Schiraldi M, Raucci A, Munoz LM, Livoti E, Celona B, Venereau $\mathrm{E}$, et al. HMGB1 promotes recruitment of inflammatory cells to damaged tissues by forming a complex with CXCL12 and signaling via CXCR4. J Exp Med. 2012;209:551-63.

10. Sorci G, Riuzzi F, Giambanco I, Donato R. RAGE in tissue homeostasis, repair and regeneration. Biochim Biophys Acta. 2013;1833:101-9.

11. Palumbo R, Sampaolesi M, De Marchis F, Tonlorenzi R, Colombetti S, Mondino A, et al. Extracellular HMGB1, a signal of tissue damage, induces mesoangioblast migration and proliferation. J Cell Biol. 2004;164:441-9.

12. Sims GP, Rowe DC, Rietdijk ST, Herbst R, Coyle AJ. HMGB1 and RAGE in inflammation and cancer. Annu Rev Immunol. 2010;28:367-88.

13. Greco R, Amantea D, Mangione AS, Petrelli F, Gentile R, Nappi $\mathrm{G}$, et al. Modulation of RAGE isoforms expression in the brain and plasma of rats exposed to transient focal cerebral ischemia. Neurochem Res. 2012;37:1508-16.

14. Kim SJ, Ryu MJ, Han J, Jang Y, Kim J, Lee MJ, et al. Activation of the HMGB1-RAGE axis upregulates TH expression in dopaminergic neurons via JNK phosphorylation. Biochem Biophys Res Comm. 2017;493:358-64.

15. Son JH, Chun HS, Joh TH, Cho S, Conti B, Lee JW. Neuroprotection and neuronal differentiation studies using substantia nigra dopaminergic cells derived from transgenic mouse embryos. J Neurosci. 1999;19:10-20.

16. Jackson-Lewis V, Przedborski S. Protocol for the MPTP mouse model of Parkinson's disease. Nature Protoc. 2007;2:141-51. 
17. Bradford MM. A rapid and sensitive method for the quantitation of microgram quantities of protein utilizing the principle of protein-dye binding. Anal Biochem. 1976;72:248-54.

18. Luk KC, Kehm VM, Zhang B, O'Brien P, Trojanowski JQ, Lee VM. Intracerebral inoculation of pathological alpha-synuclein initiates a rapidly progressive neurodegenerative alphasynucleinopathy in mice. J Exp Med. 2012;209:975-86.

19. Oh MS, Hong SJ, Huh Y, Kim KS. Expression of transgenes in midbrain dopamine neurons using the tyrosine hydroxylase promoter. Gene Ther. 2009;16:437-40.

20. Muramatsu Y, Kurosaki R, Watanabe H, Michimata M, Matsubara M, Imai $\mathrm{Y}$, et al. Cerebral alterations in a MPTP-mouse model of Parkinson's disease-an immunocytochemical study. J Neural Transm (Vienna). 2003;110:1129-44.

21. Jackson-Lewis V, Jakowec M, Burke RE, Przedborski S. Time course and morphology of dopaminergic neuronal death caused by the neurotoxin 1-methyl-4-phenyl-1,2,3,6-tetrahydropyridine. Neurodegeneration. 1995;4:257-69.

22. Jakel S, Dimou L. Glial cells and their function in the adult brain: a journey through the history of their ablation. Front Cell Neurosci. 2017;11:24

23. Sasaki T, Liu K, Agari T, Yasuhara T, Morimoto J, Okazaki M, et al. Anti-high mobility group box 1 antibody exerts neuroprotection in a rat model of Parkinson's disease. Exp Neurol. 2016;275(Pt 1):220-31.

24. Schmidt AM, Yan SD, Yan SF, Stern DM. The multiligand receptor RAGE as a progression factor amplifying immune and inflammatory responses. J Clin Invest. 2001;108:949-55.

25. Da Cunha C, Wietzikoski S, Wietzikoski EC, Miyoshi E, Ferro MM, Anselmo-Franci JA, et al. Evidence for the substantia nigra pars compacta as an essential component of a memory system independent of the hippocampal memory system. Neurobiol Learn Mem. 2003;79:236-42.

26. Shih AY, Fernandes HB, Choi FY, Kozoriz MG, Liu Y, Li P, et al. Policing the police: astrocytes modulate microglial activation. J Neurosci. 2006;26:3887-8.

27. Reynolds AD, Stone DK, Mosley RL, Gendelman HE. Proteomic studies of nitrated alpha-synuclein microglia regulation by CD4+ CD25+T cells. J Proteome Res. 2009;8:3497-511.

28. Chung YC, Kim SR, Jin BK. Paroxetine prevents loss of nigrostriatal dopaminergic neurons by inhibiting brain inflammation and oxidative stress in an experimental model of Parkinson's disease. J Immunol. 2010;185:1230-7.

29. Chao Y, Wong SC, Tan EK. Evidence of inflammatory system involvement in Parkinson's disease. BioMed Res Int. 2014;2014:308654.

30. Kim C, Lee HJ, Masliah E, Lee SJ. Non-cell-autonomous neurotoxicity of alpha-synuclein through microglial toll-like receptor 2. Exp Neurobiol. 2016;25:113-9.

31. Dawson TM. Non-autonomous cell death in Parkinson's disease. Lancet Neurol. 2008;7:474-5.

32. Wilhelmsson U, Bushong EA, Price DL, Smarr BL, Phung V, Terada M, et al. Redefining the concept of reactive astrocytes as cells that remain within their unique domains upon reaction to injury. Proc Nat Acad Sci USA. 2006;103:17513-8.

33. Srikrishna G, Freeze HH. Endogenous damage-associated molecular pattern molecules at the crossroads of inflammation and cancer. Neoplasia. 2009;11:615-28.

34. Serrano A, Donno C, Giannetti S, Peric M, Andjus P, D'Ambrosi $\mathrm{N}$, et al. The astrocytic S100B protein with its receptor RAGE is aberrantly expressed in SOD1(G93A) models, and its inhibition decreases the expression of proinflammatory genes. Mediators Inflamm. 2017;2017:1626204.

35. Mrak RE, Sheng JG, Griffin WS. Correlation of astrocytic S100 beta expression with dystrophic neurites in amyloid plaques of Alzheimer's disease. J Neuropath Exp Neurol. 1996;55:273-9.

36. Rothermundt M, Peters M, Prehn JH, Arolt V. S100B in brain damage and neurodegeneration. Microsc Res Tech. 2003;60:614-32.

37. Sathe K, Maetzler W, Lang JD, Mounsey RB, Fleckenstein C, Martin HL, et al. S100B is increased in Parkinson's disease and ablation protects against MPTP-induced toxicity through the RAGE and TNF-alpha pathway. Brain. 2012;135(Pt 11):3336-47.

38. Fuller RW, Hemrick-Luecke SK, Perry KW. Tissue concentrations of MPTP and MPP + in relation to catecholamine depletion after the oral or subcutaneous administration of MPTP to mice. Life Sci. 1989;45:2077-83.

39. Saporito MS, Thomas BA, Scott RW. MPTP activates c-Jun NH (2)-terminal kinase (JNK) and its upstream regulatory kinase MKK4 in nigrostriatal neurons in vivo. $\mathrm{J}$ Neurochem. 2000;75:1200-8.

40. Ramasamy R, Yan SF, Herold K, Clynes R, Schmidt AM. Receptor for advanced glycation end products: fundamental roles in the inflammatory response: winding the way to the pathogenesis of endothelial dysfunction and atherosclerosis. Ann NY Acad Sci. 2008;1126:7-13.

41. Teismann P, Sathe K, Bierhaus A, Leng L, Martin HL, Bucala R, et al. Receptor for advanced glycation endproducts (RAGE) deficiency protects against MPTP toxicity. Neurobiol Aging. 2012;33:2478-90.

42. Huttunen HJ, Fages C, Rauvala H. Receptor for advanced glycation end products (RAGE)-mediated neurite outgrowth and activation of NF-kappaB require the cytoplasmic domain of the receptor but different downstream signaling pathways. J Biol Chem. 1999;274:19919-24.

43. Taguchi A, Blood DC, del Toro G, Canet A, Lee DC, Qu W, et al. Blockade of RAGE-amphoterin signalling suppresses tumour growth and metastases. Nature. 2000;405:354-60.

44. Wu X, Mi Y, Yang H, Hu A, Zhang Q, Shang C. The activation of HMGB1 as a progression factor on inflammation response in normal human bronchial epithelial cells through RAGE/JNK/NFkappaB pathway. Mol Cell Biochem. 2013;380:249-57.

45. Brochard V, Combadiere B, Prigent A, Laouar Y, Perrin A, Beray-Berthat $\mathrm{V}$, et al. Infiltration of CD4+ lymphocytes into the brain contributes to neurodegeneration in a mouse model of Parkinson disease. J Clin Invest. 2009;119:182-92.

46. Sun Y, Chen H, Dai J, Wan Z, Xiong P, Xu Y, et al. Glycyrrhizin protects mice against experimental autoimmune encephalomyelitis by inhibiting high-mobility group Box 1 (HMGB1) expression and neuronal HMGB 1 release. Frontiers Immunol. 2018;9:1518.

47. Aumann TD, Egan K, Lim J, Boon WC, Bye CR, Chua HK, et al. Neuronal activity regulates expression of tyrosine hydroxylase in adult mouse substantia nigra pars compacta neurons. J Neurochem. 2011;116:646-58.

48. Yan HQ, Ma X, Chen X, Li Y, Shao L, Dixon CE. Delayed increase of tyrosine hydroxylase expression in rat nigrostriatal system after traumatic brain injury. Brain Res. 2007;1134:171-9. 